\title{
Wallet Databases with Observers
}

\author{
(Extended Abstract)
}

\author{
David Chaum \\ CWI \\ The Netherlands
}

\author{
Torben Pryds Pedersen* \\ Aarhus University \\ Denmark
}

\begin{abstract}
Previously there have been essentially only two models for computers that people can use to handle ordinary consumer transactions: (1) the tamper-proof module, such as a smart card, that the person cannot modify or probe; and (2) the personal workstation whose inner working is totally under control of the individual. The first part of this article argues that a particular combination of these two kinds of mechanism can overcome the limitations of each alone, providing both security and correctness for organizations as well as privacy and even anonymity for individuals.

Then it is shown how this combined device. called a wallet, can carry a database containing personal information. The construction presented ensures that no single part of the device (i.e. neither the iampe-proof part nor the workstation) can learn the contents of the database - this information can only be recovered by the two parts together.
\end{abstract}

\section{Introduction}

In this paper we shall be concerned with a general system consisting of a number of individuals and organizations. Each individual has a small database with (personal) information (for example credentials), and the purpose of a transaction is to either update this database (obtain a new credential) or read some information in it (show a credential). For such a system it is important that the data in the database are correct:

- The organizations want to be sure that the contents of each database corresponds to what they have written in it.

- The individuals want to be sure that the organizations only store correct information in the database. and that they can only read and update those parts of the database that they are entitled to.

- Research partly done while visiting CWI 
Another basic requirement is that it should be possible for the individuals to participate anonymously in certain transactions. If, for example, the database contains medical information, which is needed in an investigation of a particular disease, the owner might require anonymity in order to participate in this investigation. There are, however, many other kinds of transactions, such as financial transactions, in which the issue of privacy is essential as well.

Section 2 argues that the electronic wallet is well suited for this scenario. An electronic wallet consists of two parts:

- A small, hand-held computer controlled by the user-denoted by $C$, for "computer"; and

- A tamper-proof module issued by the organizations-denoted by $T$, for "tamperproof".

These two parts are arranged in such a way that $T$ can only talk with $C$ and not the outside world. This might be achieved by embedding $T$ inside $C$. All communication with organizations is via $C$. It is essential that there is no "alternative way" that $T$ can send messages to or receive messages from the outside world.

In the second part of the paper practical protocols are presented. First a new blind signature technique is presented in Section 3. Then Section 4 shows how, using the blind signatures, $T$ can get a certified public key, which it can use to sign messages and thereby authenticate the actions taken by $C$. Then Section 5 presents the database protocols. In particular, it is shown how $T$ can validate the information sent from the wallet without even knowing the contents of the database.

\section{Possible Settings}

This section discusses the advantages and disadvantages of different devices for use by individuals in a system including users and organizations, as described above. We shall primarily be concerned with how well the various alternatives support the requirements of correctuess and privacy.

\subsection{Correctness and Privacy}

Correctness basically means that the data stored in a person's database can only be read or updated by the organizations/individuals that have permission to do so (according to some initially agreed rules). Note that these rules could say that a person is not allowed to change (parts of) his own database, and they could even (in extreme situations) specify that the user may not read parts of the database.

The terms positive credential and negative credential will be used to denotc information in the database, which is to the advantage and disadvantage of the person, respectively. A bad criminal record is an example of a negative credential. The user may want to delete negative credential in the database, but this should, of course, be infeasible. 
By one-show credential we mean a credential that the individual is allowed to show only once. Electronic money, which may be spent only once, is a typical example of a one-show credential.

While correctness is the most important requirement for organizations, privacy might be the important issue for individuals (at least in some situations), and it is essential for general acceptance of the system. We distinguish three levels of privacy:

- Pure trust:

Information about the individual may be revealed during a transaction-the individual cannot do anything to enhance his privacy, but must trust the organization to maintain it.

- Computational privacy:

If the individual follows the prescribed protocols, the organization cannot learn anything about him unless it can make a computation assumed to be infeasible.

- Unconditional privacy:

If the individual follows the prescribed protocols, even an all powerful organization cannot learn anything extra about him.

\subsection{Possible Approaches}

We now analyze how two very different devices meet the demands of correctness and privacy outlined above. We first consider a device trusted completely by the individuals and then a tamper-proof device issued by the organizations (or an issuing center trusted by the organizations). This analysis then leads to the definition of electronic wallets.

\section{Computer alone}

First consider the situation where the user just has a computer, which he controls completely. In particular, he can delete or change any part of the memory, and he determines all messages which the device sends to the outside world.

Using the techniques of [Cha84] and [CF V90? it is possible to obtain unconditional privacy in this scenario in an efficient way. However, this setting makes it very difficult for the organizations to prevent users from deleting negative credentials or using one-show credentials more than once.

For example, in the case of an off-line electronic payments system, it is only known how to catch cheaters, who spend copies of the same electronic coin more than once, "after the fact". This method furthermore requires a large central database in which all valid coins are collected and compared (see [CF V90]), but this only has to be done periodically.

In short, this setting can give unconditional privacy. whereas no really efficient method for correctness is known.

\section{Tamper-proof only}

In this setting each individual has a tamper-proof module (packaged as a smart-card, for instance) issued by the organizations. Hence the organizations trust the correctness of 
the messages sent by the card, whereas the user does not even know which messages are bcing sent.

This approach gives correctness quite easily, because the tamper-proof part has to be broken in order to compromise the system. Furthermore, if cryptographic techniques are added it is sometimes possible to make systems in which cheating requires breaking the tamper resistant part and the cryptographic methods. Off-line electronic cash is an example of such a system.

If a tamper-proof unit is used to store negative credentials, the owner can delete these by destroying or throwing away the card. However, in this way he will also delete the positive credentials and, furthermore, the organizations will detect it the next time they need the card. In order to recover from such intentional as well as accidental losses of credentials, the system can have a back up facility for recovering such lost credentials. Hence, this approach can provide a very high degree of correctness.

The disadvantage of using the tamper-proof unit alone is that it only provides a low level of privacy, as the user has no control over the messages sent from the card. Therefore the card can (in principle) send any message that it likes during a transaction (e.g. the identity of the user). Hence, it can only give pure trust.

\section{Electronic Wallets}

The above analysis of two extreme settings shows that neither a user controlled computer nor a tamper-proof device alone can give sufficiently efficient and secure solutions. Electronic wallets can be thought of as a way to obtain the benefits of both approaches by a suitable combination.

Since no device that allows a tamper-proof device to communicate directly with the organizations can give a higher level of privacy than pure trust. the device must be constructed in such a way that the tamper-proof device cannot send messages to the organizations.

Thus the device should consist of a user controlled computer, $C$. with a tamper-proof unit, $T$, (sometimes called an observer). which on behalf of the organizations ensures that $C$ cannot deviate from the prescribed protocols or change any information in its database. The electronic wallet is the simplest such device as it only has a single such observer $(T)$. It might be useful (for example in order to make fault recovery easier) to have more than one observer, but such an approach does not seem to add significantly more power to the wallet.

Note, that $C$ can freely communicate with the outside world without the knowledge of $T$, but the honest organizations will only accept messages which are approved by $T$.

The rest of this paper presents protocols, which show how $T$ can control the actions of $C$. The fact that $T$ may not communicate directly with organizations means that these protocols must be secure against

- Infiow:

No matter how $T$ and the organization deviate from the prescribed protocol, if $C$ follows the protocol, the organization cannot send any extra (subliminal) information to $T$. 
- Outflow:

No matter how $T$ and the organization deviate from the prescribed protocol. if $C$ follows the protocol, $T$ cannot send any extra (subliminal) information to the organization.

This means that even if the organization places a malicious observer in the wallet, there is no way that it can send back any information about the owner.

If all protocols are secure against outflow, then the security against inflow is not that significant, because $T$ cannot tell other organizations what it learns. However, if it is important that $T$ does not reveal any secrets in case it is returned to the organizations, the protocols must be secure against inflow as well.

\section{The Signature Scheme}

This section presents the signature scheme which will be used in this paper. The notation is introduced, the basic signature scheme is described, and it is shown how it can be used in wallets. Then it is shown how to make blind signatures.

\subsection{Notation}

Let $q$ be a prime. The protocols to be presented work for any group, $G_{q}$ of order $q$. As an example of such a group we consider another prime, $p$, such that $q$ divides $p-1$, and define $G_{q}$ as the unique subgroup of $Z_{p}^{*}$ of order $q$. The element $g \in G_{q}$ will always be a generator of $G_{q}$. It will be assumed that all parties know $p, q$ and $g$.

The discrete logarithm of $h \in G_{q}$ with respect to $g$ is denoted by $\log _{g} h$, and the number of bits of an integer, $x$, will be denoted $|x|$.

\subsection{The Basic Scheme}

This subsection presents the signature scheme which will be used in the following protocols.

The public key of the scheme is

$$
(p, q, g, h)
$$

where $h \in G_{q} \backslash\{1\}$ and the corresponding secret key is $x=\log _{g} h$.

Let $m \in G_{q}$ be a message. The signature on $m$ consists of $z=m^{x}$ plus a proof that

$$
\log _{g} h=\log _{m} z \text {. }
$$

Given $m$ and $z$, consider the following protocol:

1. The prover chooses $s \in \mathbb{Z}_{q}$ at random and computes $(a, b)=\left(g^{s}, m^{s}\right)$. This pair is sent to the verifier.

2. The verifier chooses a random challenge $c \in Z_{q}$ and sends it to the prover. 
3. The prover sends back $r=s+c x$.

4. The verifier accepts the proof if

$$
g^{r}=a h^{c} \quad \text { and } \quad m^{r}=b z^{c} .
$$

If the prover can send correct responses $r_{2}$ and $r_{2}$ to two different challenges, $c_{1}$ and $c_{2}$ then

$$
g^{r_{1}-r_{2}}=h^{c_{1}-c_{2}} \text { and } m^{r_{1}-r_{2}}=z^{c_{1}-r_{2}} \text {, }
$$

and hence

$$
\log _{s} h=\log _{m} z=\frac{c_{1}-c_{2}}{r_{1}-r_{2}}
$$

since $c_{1} \neq c_{2} \bmod q$ implies that $r_{1} \neq r_{2} \bmod q$. Now let $H$ be a one-way hash function (as in the Fiat-Shamir schemc, see [FS8T]). Given this function and the above protocol the signature on $m$ is

$$
\sigma(m)=(z, a, b, r)
$$

It is correct if $c=H(m, z, a, b)$ and

$$
g^{r}=a h^{c} \text { and } \quad m^{r}=b z^{c} \text {. }
$$

Hence, a signature on a $|q|$ bits message is $|q|+3|p|$ bits long.

Now consider attempts to forge signatures given only the public key. If $H$ has the property that it is as difficult to convince a verifier, who chooses $c:=H(m, z, a, b)$, as a verifier who chooses the challenge at random ( $H$ is like a random oracle), it is not feasible to make signatures without knowing $x$.

Furthermore, it does not seem to belp a forger to execute the proof that $\log _{g} h=\log _{m} z$ with the signer for the following reason. Consider the modification of the proof system in which the challenge. $c$, is chosen from a subset $A \subseteq \mathbb{Z}_{q}$ instead of $\mathbb{Z}_{q}$. For any such subset an execution of this modified scheme can be simulated perfectly in expected time $O(|A|)$. In particular this simulation is feasible if $|A|$ is polynomial in $|q|$. It is an open question to prove that executions of the protocol are secure, when $A$ equals $\mathbb{Z}_{q}$, but we conjecture that no matter which $c \in Z_{q}$ is chosen as challenge, the signer reveals no other information than the fact that $\log _{g} h$ equals $\log _{m} z$.

Finally remains the possibility that a forger can construct a false signature by combining various given signatures $\left(m_{i}, \sigma_{i}\right)$, where the forger has chosen $m_{i}$ adaptively (see [GMR88]). If $z_{i}=m_{i}^{z}$ then

$$
z_{1} z_{2}=\left(m_{1} m_{2}\right)^{x} \text {. }
$$

Hence there is a multiplicative relation which might be useful for a forger. However: the use of $H$ should prevent the forger from combining different signatures into a new signature.

\subsection{Signatures by $T$}

This section shows how the above signature scheme can be used by the tamper-proof device $T$ in a wallet. The problem, that we have to deal with, is that $T$ cannot be allowed 
to choose $a$ and $b$ alone, as it can encode some information in these two numbers. We therefore generate these two numbers using a coin-flipping protocol. If $T$ has a public key $\left(p, q, g, h_{T}\right)$ and a corresponding secret key $x_{T}=\log _{3} h_{T}$ it can $\operatorname{sign}$ a message $m \in G_{q}$ as follows:

1. $C$ chooses $s_{0} \in \mathbb{Z}_{q}$ and $t_{0} \in \mathbb{Z}_{q}$ at random and sends $\alpha:=g^{s_{0}} h_{T}^{t_{0}}$ to $T$ (a commitment to $s_{0}$ ).

2. $T$ chooses $s_{1} \in Z_{q}$ at random and sends $a_{1}:=g^{s_{1}}$ and $b_{1}:=m^{s_{1}}$ to $C$.

3. $C$ sends $\left(s_{0}, t_{0}\right)$ to $T$ and computes $a:=a_{1} g^{s_{0}}$ and $b:=b_{1} m^{s_{0}}$.

4. $T$ verifies that $\alpha$ equals $g^{s_{0}} h_{T}^{t_{0}}$ and computes $(a . b):=\left(a_{1} g^{s_{0}}, b_{3} m^{s_{0}}\right)$.

5. $T$ computes $c:=H\left(m, m^{r+}, a, b\right)$ and $r:=s_{0}+s_{1}+c x_{T} \bmod q$

The signature on $m$ is $\left(m^{x^{T}}, a . b, r\right)$.

It is not hard to see that if $C$ follows the protocol then $a$ and $b$ are uniformly distributed in $G_{q}$. Furthermore, $C$ can only open $\alpha$ as some $s_{0}^{\prime} \neq s_{0}$ if it can find $x_{T}$. Hence, if $T$ follows the protocol and $C$ does not know $x_{T}$, then $a$ and $b$ are random elements of $G_{\text {q }}$.

\section{Proposition 3.1}

The above protocol for making signatures has the following two properties:

1. If $C$ follows the protocol, then the signature is randomly distributed among the signatures on $m$ - even if a cheating $T$ has unlimited computing power.

2. If $T$ follows the protocol, then a polynomially bounded cheating $C$ learns no more than a random signature on $m$.

\section{Proof}

Both claims follow from the fact that the coin-flipping protocol in Step $1-4$ above has the following two properties:

1. $(a, b)$ is uniformly distributed among the possible pairs. if $C$ follows the protocol even if a cheating $T$ has unlimited computing power (because $\alpha$ contains no Shannon information about $s_{0}$ ).

2. A polynomially bounded $C$ can only open $\alpha$ in two different ways if it knows $\log _{g} h_{T}$.

\subsection{Blind Signatures}

To get a blind signature on the message $m$ in the above scheme one chooses a random $t \in \mathbb{Z}_{\beta}^{*}$ and asks the signer to sign $m_{0}=m^{t}$. Let $z_{0}=m_{0}{ }^{x}$. Then the signer proves that $\log _{g} h=\log _{m_{0}} z_{0}$ in such a way that the messages are blinded:

1. The signer chooses $s \in \mathbb{Z}_{q}$ at random and computes $\left(a_{0}, b_{0}\right)=\left(g^{s}, m_{0}{ }^{s}\right)$. This pair is sent to the verifier 
2. The verifier chooses $u \in \mathbb{Z}_{q}^{*}$ and $v \in Z_{\gamma}$ at random and computes

$$
a=\left(a_{0} g^{u}\right)^{u} \text { and } \quad b=\left(b_{0}^{1 / t} m^{v}\right)^{u} \text {. }
$$

(If both parties follow the protocal $a=\left(g^{s+v}\right)^{u}$ and $b=\left(m^{s+v}\right)^{x}$.) Then the verifier computes $z=z_{0}^{1 / t}$, the challenge $c=H(m, z, a, b)$ and the blinded challenge $c_{0}=$ $c / u \bmod q$. The verifier sends $c_{0}$ to the signer.

3. The signer sends back $r_{0}=s+c_{0} x$.

4. The verifier accepts if

$$
g^{r_{0}}=a_{0} h^{c_{0}} \quad \text { and } \quad m_{0}^{r_{0}}=b_{0} z_{0}^{c_{0}} \text {. }
$$

The verifier computes $r=\left(r_{0}+v\right) u \bmod q$ and

$$
\sigma=(z, a, b, r)
$$

\section{Proposition 3.2}

$\sigma$ is a correct signature on $m$, if the verifier accepts in the above protocol.

\section{Proof}

Let $c=H(m, z, a, b)$. We have to prove that

$$
g^{r}=a h^{c} \text { and } m^{r}=b z^{c} \text {. }
$$

The first equality follows from

$$
g^{\top}=\left(g^{r_{0}} g^{u}\right)^{u}=\left(a_{0} h^{c_{0}} g^{v}\right)^{t}=\left(a_{0} g^{u}\right)^{u} h^{c_{0} u}=a h^{c}
$$

and the second from

$$
\begin{aligned}
m^{r} & =m^{u t_{0}} m^{u v} \\
& =\left(m_{0}^{1 / t}\right)^{u r_{0}} m^{v u} \\
& =\left(m_{0}^{r_{0}}\right)^{u / t} m^{v u} \\
& =\left(b_{0} z_{0}^{c_{0}}\right)^{u / t} m^{v u t} \\
& =\left(b_{0}^{1 / t} m^{v}\right)^{u} z_{0}^{u c_{0} / t} \\
& =b z^{c} .
\end{aligned}
$$

\section{Proposition $\mathbf{3 . 3}$}

The signer gets no information about $m$ and $\sigma$ if the receiver follows the protocol. 


\section{Proof}

We will show that for all $m, z, a, b$ and $r$ such that

$$
\begin{aligned}
g^{r} & =a h^{2} \\
m^{r} & =b z^{c} \\
c & =H(m, z, a, b)
\end{aligned}
$$

and for all $m_{0}, z_{0}, a_{0}, b_{0}, c_{0}$ and $r_{0}$ such that

$$
\begin{aligned}
g^{+0} & =a_{0} h^{c_{0}} \\
m_{0}^{\prime 0} & =b_{0} z_{0}^{c_{0}}
\end{aligned}
$$

there is exactly one set of values of $t, u$ and $v$ such that the signer sees $\left(m_{0}, z_{0}, o_{0}, b_{0}, c_{0}, r_{0}\right)$, when making the signature $\sigma$ on $\mathrm{m}$. In other words, that there is exactly one set of values of $t, u$ and $v$ such that

$$
\begin{aligned}
m & =m_{0}^{1 / t} \\
a & =\left(a_{0} g^{v}\right)^{4} \\
b & =\left(b_{0}^{1 / t} m^{v}\right)^{u} \\
c & =c_{0} u \\
r & =\left(r_{0}+v\right) u .
\end{aligned}
$$

First, $m$ and $m_{0}$ determine $t$ as

$$
m_{0}=m^{t} \Longleftrightarrow t=\log _{m} m_{0} .
$$

Secondly, $u$ and $v$ are determined by $c . c_{0} . r$ and $r_{0}$ as

$$
u=\frac{c}{c_{0}} \text { and } \quad v=\frac{c_{0}}{c} r-r_{0} .
$$

Thus we just need to show that these values of $t, u$ and $v$ satisfy

$$
a=\left(a_{0} g^{v}\right)^{u} \text { and } b=\left(b_{0}^{1 / t} m^{v}\right)^{u} \text {. }
$$

In doing this it can be assumed that $z_{0}=m_{0}^{x}$ and $z=m^{x}$, because the signer actually proves that $z_{0}$ equals $m_{0}^{x}$ when making a blind signature. Hence $m_{0}=m^{t}$ implies that

$$
\tilde{z}_{0}=z^{t} \text {. }
$$

The first equality is proven as follows

$$
a=g^{r} h^{-c}=g^{\left(r_{0}-u\right) u} h^{-u c o}=\left(g^{r_{0}} g^{v} h^{-c o}\right)^{u}=\left(a_{0} g^{v}\right)^{u} .
$$

The second equality follows by similar rewritings:

$$
\begin{aligned}
b & =m^{r} z^{-c} \\
& =m^{(r 0+v)^{u}} z^{-c_{0} u} \\
& =\left(m^{r o} m^{v} z^{-c_{0}}\right)^{u} \\
& =\left(\left(m_{0}^{1 / t}\right)^{r_{0}} m^{v}\left(z_{0}^{1 / t}\right)^{-c_{0}}\right)^{u} \\
& =\left(\left(m_{0}^{r_{0}} z_{0}^{-c_{0}}\right)^{1 / t} m^{v}\right)^{u} \\
& =\left(b_{0}^{1 / t} m^{v}\right)^{u} .
\end{aligned}
$$


This completes the proof.

Hence, this signature scheme allows the receiver to obtain blind signatures. In particular it is possible for the receiver to get a signature on any message that he chooses. In order to avoid this problem in the application to wallets, the organization only signs a blinded message if the challenge is signed by $T$. The resulting scheme is presented in the next subsection.

\subsection{Blind Signatures in Wallets}

We assume that a center $Z$ is the signer. The public key of $Z$ is $h_{Z}$ and the secret key is $x_{Z}=\log _{g} h_{Z}$.

1. $C$ chooses the blinding factor $t \in \mathbb{Z}_{q}^{*}$ at random and sends $m_{0}:=m^{2}$ to $Z$.

2. $Z$ and $C$ choose $a_{0}$ and $b_{0}$ using a coin-fipping (as in Section 3.3) protocol, such that only $Z$ knows $s=\log _{g} a_{0}=\log _{m_{0}} b_{0}$.

3. $Z$ computes $z_{0}:=m_{0}^{x z}$ and sends it to $C$.

4. $C$ computes $z:=z_{0}^{1 / t}$ and chooses $u$ and $v$ at random. Then it sends $\left(a_{0}, b_{0}, z, u, v, t\right)$ to $T$.

5. Both $T$ and $C$ can then compute $a:=\left(a_{0} g^{v}\right)^{u}, b:=\left(b_{0}^{1 / t} m^{v}\right)^{u}, c:=H(m, z, a, b)$ and $c_{0}:=c / u$. $T$ signs $c_{0}$ and sends it to $C$.

6. $G$ verifies the signature before sending the challenge and the signature to $Z$.

7. From now on the protocol for constructing and verifying blind signatures is followed. Hence $Z$ computes the response, $r_{0}$ and sends it to $C$. $C$ verifies this response before forwarding it to $T$. Finally $T$ unblinds $r_{0}$ and verifies the signature.

\section{Theorem 3.4}

If $C$ follows the protocol then

1. $Z$ gets no information about the signature on $m$.

2. $T$ sends no information to $Z$ except a random signature on $c_{0}$.

3. $Z$ sends no information to $T$ except $\approx$.

\section{Proof}

Assume that $C$ follows the protocol.

1. $Z$ sees messages with the same distribution as in the original protocol for making blind signatures - except that $Z$ cannot choose $\left(a_{0}, b_{0}\right)$ freely anymore. But this pair is chosen at random. Hence this property follows from Proposition 3.3. 
2. The only information, which originates from $T$ is the signature on $c_{0}$. However, Proposition 3.1 implies that this signature is randomly chosen among the possible signatures.

3. $T$ sees the following messages from $Z$ :

$$
\left(a_{0}, b_{0}\right), z_{0}^{1 / t} \text { and } r_{0}
$$

and $T$ receives $u, v$ and $t$ from $C$. Here $\left(a_{0}, b_{0}\right)$ is uniformly distributed (by the same argument as in the proof of Proposition 3.1), and $r_{0}$ is uniquely determined. Hence, $Z$ can only send information to $T$ via $z$.

Note that if $Z$ does not compute $z_{0}$ as $m_{0}^{\tau z}$ then $C$ will discover it. Thus, it is impossible for $Z$ to send information to $T$ without being detected. However, as we shall see in the next section even this possibility of inflow is eliminated in our application of the protocol.

We now look at the security of the protocol and assume that $T$ and $Z$ both follow the protocol. It will be argued that if the basic signature scheme is secure, and if $T$ 's signatures cannot be faked, then no matter what a polynomially bounded $\tilde{C}$ does; it learns no more than a random signature on $m$.

As $\bar{C}$ cannot forge $T$ 's signatures, it can be assumed that $c_{0}$ is computed as $c_{0}:=$ $H(m, \tilde{z}, \tilde{a}, \tilde{b})$, where $\tilde{C}$ can choose $\tilde{z}, \tilde{a}$ and $\tilde{b}$, but not $m$. By the assumption about $H$ this means that $\tilde{C}$ cannot control the value of $c_{0}\left(\tilde{C}\right.$ cannot force $c_{0}$ to be any particular. value, except by trying different values for $\tilde{z}, \bar{a}$ and $\tilde{b}$ and hoping they will give a "good" value of $c_{0}$ ). Thus $\tilde{C}$ does not seem be better off in this situation than when it just gets a "normal" signature from the signer.

\section{Obtaining a Pseudonym}

This section shows how the wallet can get a public key, which is signed by a key authentication center. The signature on the public key will be called a validator. This protocol has the property that neither the center nor any other unlimited powerful organization can link the identity of the user to the public key (or its validator).

Combining this result with Section 3.3 gives a method for $T$ to sign messages without revealing any information at all about the owner of the wallet. This provides a method for $T$ to validate the messages, which $C$ sends to the outside world, without revealing anything about the identity of the user; these messages are only accepted by the organizations if they are signed properly (by $T$ ).

We now show how $T$ can generate a secret key $x \in \mathbb{Z}_{q}^{*}$ and obtain a certificate on the corresponding public key $h=g^{x} \bmod p$. In order to get started, it is assumed that each $T$ is born with a secret key, $x_{T}$, and a corresponding public key, $h_{T}$, to the signature scheme described in Section 3.3. These signatures can be traced to $T$ (and hence to the individual), and they are therefore only used in an initial step where $T$ gets a validated key from a key authentication center $(Z)$. The center issues validators using the blind signature scheme from the previous section with secret key $x_{Z}$ and public key $h_{Z}$. 
The basic idea of the protocol for issuing validators is that $C$ and $T$ first execute a coin-flipping protocol in order to choose a secret key, $x$, which only $T$ learns. The corresponding public key is denoted by $h$. Then $C$ chooses a blinding factor, $t \in \mathbb{Z}_{q}^{*}$, and $C$ signs the blinded public key $\left(h_{1}=h^{t}\right)$. Note, that in the process of making the blind signature, $T$ has to sign a challenge computed as $H\left(h, h^{x z}, a, b\right)$. This signature guarantees to $Z$ that it validates a public key which is accepted by $T$. There is no need that $T$ signs $h_{1}$ before $Z$ starts making the blind signature, because before $Z$ computes the response, it only produces random messages, which a cheating $C$ could have produced by itself. In more detail the protocol goes like this:

1. $C$ chooses $y_{0} \in \mathbb{Z}_{q}^{*}$ at random and sends a commitment to $y_{0}$ to $T$.

2. $T$ chooses $y_{1} \in \mathbb{Z}_{q}^{*}$ at random and sends $h_{0}:=g^{y_{1}}$ to $C$.

3. $C$ opens the commitment and sends $y_{0}$ to $T$.

4. $T$ and $C$ compute $h:=h_{0}^{y_{0}}$, and $T$ computes the secret key $x:=y_{0} y_{1} \bmod q$.

5. $T$ computes $z:=h_{z}^{z}$ and sends it to $C$.

6. $C$ chooses $t \in Z_{3}$ at random and sends $h_{1}:=h^{t}$ to $Z$.

7. $Z$ makes a blind signature on $h$ by signing $h_{1}$ as follows:

(a) $Z$ computes $z_{0}:=h_{1}^{x z}$. Then $Z$ and $C$ choose $\left(a_{0}, b_{0}\right):=\left(g^{s_{0}}, h_{1}^{s_{0}}\right)$ at random such that only $Z$ knows so, whereas both know $a_{0}$ and $b_{0}$. $Z$ sends $z_{0}$ to $C$.

(b) $C$ first verifies that $z_{0}=z^{\prime}$, and then it chooses $u \in \mathbb{Z}_{q}^{*}$ and $v \in \mathbb{Z}_{q}$ at random and computes

$$
a:=\left(a_{0} g^{2}\right)^{u} \text { and } b:=\left(b_{0}^{1 / t} h^{2}\right)^{i t} \text {. }
$$

$C$ then sends $u, v, t$ and $\left(a_{0}, b_{0}\right)$ to $T$.

(c) $T$ computes the pair ( $a . b$ ) just as $C$ did, the challenge $c:=H(h, z, a, b)$, and $c_{0}:=c / u \bmod q$. Then it signs $c_{0}$ using $x_{I}$ (with help from $C$ ) and sends the signature to $C$.

(d) $C$ computes $c:=H(h, z, a, b), c_{0}:=c / u$, and verifies the signature. $C$ then forwards $c_{0}$ and the signature to $Z$.

(e) $Z$ verifies the signature on $c_{0}$ and computes $r_{0}:=s_{0}+c_{0} s_{Z} \bmod q$.

(f) $C$ verifies that

$$
g^{r_{0}}=a_{0} h_{Z}^{c 0} \quad \text { and } \quad h_{i}^{r_{0}}=b_{0} z_{0}^{\infty}
$$

and computes $r=\left(r_{0}+v\right) u \bmod q$. Then $C$ forwards $r_{0}$ to $T$.

(g) $T$ cornputes $r:=\left(r_{0}+v\right) u \bmod q$ and verifies that:

$$
g^{r}=a h_{Z}^{c} \quad \text { and } \quad h^{r}=b i^{5} \text {. }
$$

\section{Theorem 4.1}

This protocol satisfies: 
1. If $T, C$ and $Z$ follow the protocoi, then $T$ gets $Z$ 's signature on $h$.

2. If $C$ follows the protocol then $Z$ gets no information about $h$ or $\sigma$. This is true even if $T$ and $Z$ have unlimited computing power.

3. If $C$ follows the protocol then $Z$ can construct all messages with the same distribution in expected polynomial time except the signature on $c_{0}$.

4. If $C$ follows the protocol, then $T$ can simulate all messages that it receives - except $r_{0}$.

5 . If the blind signature scheme is secure then a polynomially bounded $\tilde{C}$ cannot get a validated public key for which he knows the corresponding secret key.

\section{Proof}

The first three properties are straightforward to prove, and the fourth follows from Theorem 3.4 and the fact that $T$ can compute $z$ by itself. As for the last property, note that the security of the blind signature scheme means that $\tilde{C}$ can only get a signature on $h$, but $\tilde{C}$ cannot find the secret key corresponding to $h$ (i.e. $\log _{g} h$ ) unless it can compute discrete logarithms in $G_{q}$.

As $C$ can make sure that the signature on $c_{0}$ is random among all possible signatures, this theorem shows that the protocol for issuing a validated public key has no outflow. Furthermore, as $r_{0}$ is uniquely determined from the other messages the protocol protects against inflow.

\section{An Application to Databases}

This section first describes how a very simple database offering unconditional privacy as well as correctness can be constructed, and then it is shown how a database in which the information is kept secret from both $T$ and $C$ can be constructed. By similar techniques. it is also possible to construct databases in which

1. The data is known by $T$, but kept secret from $C$ : and

2. The data is known by $C$, but kept secret from $T$.

Whenever $T$ signs a message (anonymously) with respect to a public key, which is valjdated by the key authentication center, the signature will be referred to as a certified signature.

\subsection{A Simple Database}

The wallet can be used to store the personal database described in the introduction as follows:

- All information in the database is stored by $T$ and $C$. 
- Whenever an organization updates a field in the database, it sends a signed message to the wallet. $C$ verifies the signature before it updates the database and forwards the new information plus the signature to $T$. Finally $T$ verifies the signature and updates the database.

- When an organization wants to read a field in the database (or a function-value of several fields), a certified signature on the value is sent to the organization.

\subsection{Database with Hidden Information}

The implementation presented above has the property that both $T$ and $C$ know all information in the database. This could be a little dangerous for the user, because $T$ could leak all information, in case it is captured by another person, who is able to break the tamper-resistance. On the other hand, there might be certain very sensitive data in the database, which the user should not know either (or does not want to be stored in his computer).

In the following it is therefore shown how the above database can be modified such that neither $T$ nor $C$ knows the data, but $T$ is still able to control that $C$ does not change anything in the database. We shall, however, only give protocols which allow the organization to read or write a single bit in the database. The following scheme for probabilistic encryption is an important ingredient in these protocols.

\section{Probabilistic Encryption}

Let $n=p q$, where $p$ and $q$ are primes both equivalent to 3 modulo 4 . In order to encrypt a bit $b$, the committer chooses $r \in \mathbb{Z}_{n}^{*}$ at random and computes

$$
B C(n, b, r):=(-1)^{b} r^{2} \bmod n \text {. }
$$

A person knowing $p$ and $q$ can decipher a given ciphertext by determining whether it is a. quadratic residue or not. However, for a person not knowing $p$ and $q$ this is presumably infeasible.

Let $n_{1}$ and $n_{2}$ be two different moduli as above, and let $\beta_{1}=(-1)^{b} r_{1}^{2} \bmod n_{1}$ and $\beta_{2}=(-1)^{b} r_{2}^{2} \bmod n_{2}$ be probabilistic encryptions of the same bit $b \in\{0,1\}$.

\section{Theorem 5.1}

There exists a four-round protocol with security parameter $k$ in which a person, $P$. knowing $r_{1}$ and $r_{2}$ can prove to another person, $V$, that $\beta_{1}$ and $\beta_{2}$ are in fact encryptions of the same bit. More precisely this protocol satisfies:

1. If $P$ and $V$ follow the protocol, then $V$ will accept, if $a=b$.

2. If $V$ follows the protocol and $a \neq b$, then $V$ will reject the proof with probability at least $2^{-k}$ no matter what an unlimited powerful prover does.

3. It is a proof of knowledge of $r_{1}$ and $r_{2}$.

4. It is (computationai) witness hiding (see [FS90]).

\section{Proof}

The protocol uses the cut-and-choose technique. The details are omitted here. 


\section{The Protocols}

It is assumed that each organization, $W$, has a modulus, $n_{W}$, as above, aud that $W$ can make digital signatures. Prior to the execution of the read and write protocols to be described, the following start-up protocol is executed:

1. $W$ constructs a request of the form $\left(n_{W}, o p\right.$, name, time), where op $\in\{$ read, writ $\epsilon$. name identifies the bit which $W$ wants to read or write. and time is a time-stamp. This request is signed and sent to the wallet together with certificates, which show that $n_{W}$ is a valid modulus and that the public key of $W$ (for the signature scheme) is valid.

2. $C$ verifies the request and ccrificates, and if they are legal, $C$ forwards them to $T$. In particular, $C$ verifies that time is constructed correctly so that $W$ has not encoded any information in it.

3. $T$ verifies the request and the certificates.

Whenever $T$ and $C$ sign a message in the certified signature scheme op, name, time and $n_{W}$ are included in the message. This prevents obvious frauds by $C$ in which signatures from previous executions of the same or different protocols are reused.

Furthermore, each write protocol must be immediately followed by a protocol in which $T$ sends a signed message to $W$ (through $C$ ) in which it confirms having received the required messages.

For each bit $b$ in the database, $T$ has given $C$ a commitment $3_{T}=B C\left(n_{0}, b_{T}, r_{T}\right)$ to a bit $b_{T}$, and $C$ has given $T$ a commitment $\beta_{C}=B C\left(n_{0}, b_{C}, T_{C}\right)$ to a bit $b_{C}$ such that $b=b_{T} \oplus b_{C}$. The modulus $n_{0}$ is the modulus of the organization which wrote $b$. An organization, $W$, with public modulus $n_{W}$ can read $b$ as follows

1. $T$ chooses $s_{T} \in \mathbb{Z}_{r, W}^{*}$ at random and sends $\alpha_{T}:=(-1)^{b} s_{T}^{2} \bmod n_{W}$ to $C$. $T$ proves to $C$ that $\alpha_{T}$ and $\beta_{T}$ are encryptions of the same bit.

2. $C$ chooses $s_{C} \in \mathbb{Z}_{r_{W}}^{*}$ at random and sends $\alpha_{C}:=(-1)^{t} c_{s_{C}}^{2} \bmod n_{W}$ to $T$. $C$ proves to $T$ that $\alpha_{C}$ and $\beta_{C}$ encrypt the same bit.

3. $T$ and $C$ sign $\alpha:=\alpha_{T} \alpha_{C}$ using the certified signature scheme. This signature (and $\alpha$ ) is sent to $W$ (through $C$ ).

4. $W$ verifies the signature and finds the encrypted bit by deciphering $\alpha$.

This protocol has the following properties:

- If $C$ follows the protocol: No matter what (an unlimited powerful) $T$ does, $\alpha$ is a random encryption of $b$. Furthermore, the signature on $\alpha$ does not contain any information other than the fact that a legal $T$ produced it.

- If $T$ follows the protocol, then $\alpha$ is an encryption of $b$ as long as $C$ cannot fake $T$ 's signatures (or break the tamper-proofness). 
- It does not make it easier for $T$ and/or $C$ to find $b$ unless $W$ tells them how to distinguish encryptions of 0 from encryptions of 1 modulo $n_{w}$.

The proofs of these properties are quite straightforward, and they are omitted from this extended abstract. The organization, $W$, can write a bit, $b$, in a given field in the database as follows:

1. $T$ chooses $a_{T} \in\{0,1\}$ and $r_{T} \in \mathbb{Z}_{r_{W}}^{*}$ at random and sends $\alpha_{T}:=(-1)^{a} r_{T}^{2} \bmod n_{W}$ to $C$.

2. $C$ chooses $a_{C} \in\{0,1\}$ and $s_{C} \in Z_{\pi_{W}}^{*}$ at random and sends $\alpha_{C}:=(-1)^{a c} s_{C}^{2} \bmod n_{W}$ to $T$.

3. $T$ and $C \operatorname{sign} \alpha:=\alpha_{T} \alpha_{C}$ in the certified signature scheme. This signature (and $\alpha$ ) is sent to $W$ (through $C$ ).

4. $W$ verifies the signature and finds the bit $a$ by deciphering $\alpha$.

5. $W$ and $C$ choose $r \in Z_{\eta_{W}}$ at random using a coin-flipping protocol.

6. $W$ then computes $b^{\prime}=a \oplus b$ and $\alpha_{W}:=(-1)^{b^{\prime}} \alpha r^{2}$, which it subsequently signs. $W$ sends the signature $\left(\sigma_{W}\right)$ to $C$.

7. $C$ computes $\alpha_{W}$, verifies $\sigma_{W}$ and computes $\beta_{C}:=\alpha_{W} \alpha_{I}^{-1}$ and $\beta_{T}:=\alpha_{T}$ and $r_{C}:=$ $r s_{C}$ and

$$
b_{C}:= \begin{cases}a_{C} & \text { if } \alpha_{W}=\alpha r^{2} \\ a_{C} \ominus 1 & \text { if } \alpha_{W}=-a r^{2}\end{cases}
$$

$C$ then forwards $\alpha_{W}$ and $\sigma_{W}$ to $T$.

8. $T$ verifies the signature and computes $\beta_{C}:=\alpha_{w} \alpha_{T}^{-1}$ and $\beta_{T}:=\alpha_{T}$ and lets $b_{T}=a_{T}$.

This protocol satisfies (again the proofs are omitted):

- If $T, C$ and $W$ follow the protocol then after the execution the following holds:

1. $\beta_{T}=B C\left(n_{W}, b_{T}, r_{T}\right)$

2. $\beta_{C}=B C\left(n_{W}, b_{C}, r_{C}\right)$;

3. $b=b_{T} \oplus b_{C}$.

- If $C$ cannot fake $T$ 's or $W$ 's signatures then $b \Theta b_{I}$ equals the plaintext corresponding to $\beta_{C}$.

- After the execution $b \ominus b_{C}$ equals the plaintext corresponding to $\beta_{T}$ no matter what an unlimited powerful $T$ does.

- $C$ and/or $T$ can only find $b$ if they can distinguish quadratic residues from quadratic non-residues modulo $n_{W}$. 
- If $C$ follows the protocol, then $W$ just gets a signature on a random encryption of a random bit. Similarly, $T$ just gets a vandom encryption of a random bit chosen by $W$.

In the above two protocols the amount of inflow and outflow is very limited. Note, that $W$ could have told $T$ the factorization of $n_{W}$ in advance. Hence, $T$ learns the bit. However, this does seem to be a serious problem as $W$ already knows this bit.

\section{Conclusion and Future Work}

We have argued that the electronic wallets presented here are an excellent way to store personal databases. And we have shown protocols that allow $T$ to control and validate all messages from the user to the outside world. These protocols allow $C$ to ensure that the privacy of the person is not compromised. Thcy provide organizations with security against abuse by individuals that relies on the assumption that the tamper-proofness cannot be broken and that the signatures cannot be forged.

The protocols presented do, however, have a limited kind of inflow because $T$ and $W$ see the same random values (such as those used to form the signatures). In case $T$ gets captured, these values would let organizations who could read out the contents of a captured $T$ link it to specific protocol instances. Forthcoming joint work with Stefan Brands: Ronald Cramer and Niels Ferguson shows how the need for observers and organizations to share such information can be avoided altogether.

\section{References}

[CFN90] D. Chaum, A. Fiat, and M. Naor. Untraceable electronic cash. In Adrances in Cryptology - proceedings of CRYPTO 88, Lecture Votes in Computer Science. pages $319-327$. Springer-Verlag. 1990.

[Cha84] D. Chaum. Blind signature systems. In Advances in Cryptology - proceedings of CRYPTO 83. 1984.

[FS87] A. Fiat and A. Shamir. How to prove yourself: Practical solutions to identification and signature problems. In Advances in Cryptology - proceedings of EUROCRYPT 86, Lecture Notes in Computer Science; pages 136 - 194. Springer-Verlag, 1987.

[FS90] U. Feige and A. Shamir. Witness indistinguishable and witness hiding protocols. In Proceedings of the 2:2nd Annual ACM Symposium on the Theory of Computing, pages $416-426,1990$.

[GMR88] S. Goldwasser, S. Micali, and R. L. Rivest. A digital signature scheme secure against adaptive chosen message attack. SIAM Journal on Computing, $17(2): 281$ - 308, April 1988 . 